\title{
Retraction Note: Human bone marrow-derived mesenchymal stem cell-secreted exosomes overexpressing microRNA-34a ameliorate glioblastoma development via down-regulating MYCN
}

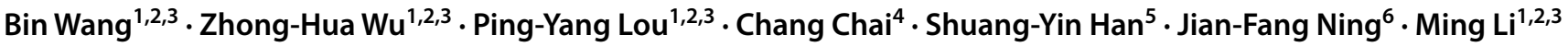 \\ (c) International Society for Cellular Oncology 2019
}

\section{Retraction Note: Cellular Oncology https://doi.org/10.1007/s13402-019-00461-z}

The Editor-in-Chief has retracted this article. After publication, the corresponding author stated that he has been unable to reproduce the targeting relationship between miR34a and MYCN in animal experiments. Additionally, the authors have been unable to provide documents confirming that ethics approval was obtained for this study. The Editorin-Chief therefore considers the data reported in this the article to be unreliable.The authors have not responded to correspondence regarding this retraction notice.

Publisher's Note Springer Nature remains neutral with regard to jurisdictional claims in published maps and institutional affiliations.
The original article can be found online at https://doi.org/10.1007/ s13402-019-00461-z.

Ming Li

liming0914@yeah.net

1 Department of Neurosurgery, Henan Province

People's Hospital, Zhengzhou University, No. 7,

Weiwu Road, Zhengzhou 450003, Henan Province,

People's Republic of China

2 Department of Neurosurgery, People's Hospital Affiliated With Zhengzhou University,

No. 7, Weiwu Road, Zhengzhou 450003, Henan Province, People's Republic of China

3 Department of Neurosurgery, People's Hospital Affiliated With Medical College of Henan University,
No. 7, Weiwu Road, Zhengzhou 450003, Henan Province, People's Republic of China

4 Department of Ophthalmology, Henan Province People's Hospital, Zhengzhou University, Zhengzhou 450003, People's Republic of China

5 Center for Translational Medicine, Henan Province People's Hospital, Zhengzhou University, Zhengzhou 450003, People's Republic of China

6 Department of Neurosurgery, University of Minnesota, Minneapolis 55455, USA 